\title{
Diagnóstico molecular da infecção por hemoplasmas em gatos domésticos naturalmente infectados da cidade de Belém, Pará ${ }^{1}$
}

\author{
Sinerey K.S. Aragão-de-Sousa², Francisco D. Sampaio-Junior ${ }^{3}$, Luciane O. Sousa ${ }^{3}$, Rafaelle \\ C. Santos ${ }^{3}$, Evonnildo C. Gonçalves ${ }^{4}$, Alessandra Scofield ${ }^{3}$ e Gustavo Góes-Cavalcante ${ }^{3 *}$
}

\begin{abstract}
Aragão-de-Sousa S.K.S., Sampaio-Junior F.D., Sousa L.O., Santos R.C., Gonçalves E.C., Scofield A. \& Góes-Cavalcante G. 2013. [Molecular diagnosis of haemoplasmas infection in naturally infected domestic cats from Belém, Pará.] Diagnóstico molecular da infecção por hemoplasmas em gatos domésticos naturalmente infectados da cidade de Belém, Pará. Pesquisa Veterinária Brasileira 33(9):1116-1120. Laboratório de Parasitologia Animal, Instituto de Medicina Veterinária, Universidade Federal do Pará, BR-316 Km 68, Castanhal, PA 68743-000, Brazil. E-mail: ggcavalcante@ufpa.br

Mycoplasma haemofelis, 'Candidatus Mycoplasma haemominutum' and 'Candidatus Mycoplasma turicensis' are the causative agent of the feline mycoplasmosis, which could cause acute or chronic anemia. The aim of this work was to determine the occurrence of hemoplamas in domestic cats from Belém, Pará. To this, 201 cats were divided into three groups: Group A were composed by 101 stray cats captured by Zoonosis Control Center, group B were composed by 62 owners healthy cats and group $C$ were composed by 38 owners cats that were suffering by some medical condition. Blood samples were collected to perform Polymerase Chain Reaction (PCR) to detect the DNA of these agents, which were sequenced and aligned. Statistical analysis was performed to detect association between the infection, the sex of the animals and experimental groups. The DNA of at least one of the hemoplasmas studied were detected in 19,9\% (40/201) of the samples, being the DNA of 'Candidatus M. haemominutum' was found in 7.96\% (16/201) of samples, M. haemofelis in $1.49 \%$ (3/201) of samples, while 'Candidatus M. turicensis' in $12.93 \%(26 / 201)$ of the samples. The DNA of these three agents was detected in cats from groups A and C, while in Group B was detected only 'Candidatus M. turicensis' and 'Candidatus M. haemominutum'. The influence of sex on hemoplasma infection was detected only between 'Candidatus $\mathrm{M}$. haemominutum' and males. These findings showed that hemoplasma circulate among domestic cats in Belém, and 'Candidatus M. turicensis' and 'Candidatus M. haemominutum' were more common than M. haemofelis, especially in stray cats.
\end{abstract}

INDEX TERMS: Mycoplasma, mycoplasmosis, PCR, domestic cats, epidemiology.

RESUMO.- Mycoplasma haemofelis, 'Candidatus Mycoplasma haemominutum' e 'Candidatus Mycoplasma turicensis' são os agentes causadores da micoplasmose felina, que podem causar anemia aguda ou crônica. 0 objetivo deste

\footnotetext{
${ }^{1}$ Recebido em 23 de julho de 2013.

Aceito para publicação em 3 de setembro de 2013.

${ }^{2}$ Hospital Veterinário, Universidade Federal Rural da Amazônia (UFRA), Av. Pres. Tancredo Neves 2501, Bairro Montese, Belém, PA 66077-901, Brasil.

${ }^{3}$ Laboratório de Parasitologia Animal, Instituto de Medicina Veterinária, Universidade Federal do Pará (UFRA), BR 316 Km 68, Castanhal, PA 68743-000, Brasil.

${ }^{4}$ Laboratório de Tecnologia Biomolecular, Instituto de Ciências Biológicas, UFPA, Rua Augusto Correa 1, Guamá, Belém, PA 66075-110. *Autor para correspondêcia: ggcavalcante@ufpa.br
}

trabalho foi determinar a ocorrência de hemoplasmas em gatos domésticos de Belém, Pará. Para isso, 201 gatos foram divididos em três grupos: Grupo A foi composto por 101 gatos de rua capturados pelo Centro de Controle de Zoonoses, o grupo B foi composto por 62 gatos domiciliados e saudáveis e o grupo $\mathrm{C}$ foi composto por 38 gatos domiciliados que apresentavam alguma afecção clínica. Foram coletadas amostras de sangue para a realização de Reação em Cadeia pela Polimerase (PCR) para detectar o DNA destes agentes, os quais foram sequenciados e alinhados. A análise estatística foi realizada para detectar a associação entre a infecção, o sexo dos animais e os grupos experimentais. O DNA de pelo menos uma das espécies de hemoplasmas pesquisados foi detectado em 19,9\% (40/201) das amostras, sendo o DNA 
de 'Candidatus M. haemominutum' encontrado em 7,96\% $(16 / 201)$ das amostras, M. haemofelis em 1,49\% (3/201) das amostras, enquanto que o DNA de 'Candidatus M. turicensis' foi detectado em $12,93 \%$ (26/201) das amostras. 0 DNA destes três agentes foi detectado em gatos dos grupos $\mathrm{A}$ e C, enquanto que no grupo B foi detectado apenas 'Candidatus M. turicensis' e 'Candidatus M. haemominutum' Foi detectada a influência do sexo sobre a infecção hemoplasmas apenas entre 'Candidatus M. haemominutum' e machos. Estes resultados mostraram que os hemoplasmas circulam entre os gatos domésticos em Belém e 'Candidatus M. turicensis' e 'Candidatus $\mathrm{M}$. haemominutum' foram mais comuns do que $M$. haemofelis, especialmente em gatos vadios.

TERMOS DE INDEXAÇÃO: Mycoplasma, micoplasmose, PCR, gatos domésticos, epidemiologia.

\section{INTRODUÇÃo}

A Micoplasmose hemotrópica felina (MHF) ou Anemia Infecciosa Felina é uma enfermidade causada pelos agentes epicelulares Mycoplasma haemofelis, 'Candidatus M. haemominutum' e/ou 'Candidatus M. turicensis' (Sykes 2003, Willi et al. 2005) que se aderem à superfície dos eritrócitos dos felídeos, levando a sua destruição pelo sistema fagocítico mononuclear (Messick 2004) e gerando um quadro de anemia hemolítica aguda ou crônica (Souza \& Almonny 2002, Page 2003).

Existem poucas informações sobre a transmissão das diferentes espécies de hemoplasmas que acometem felinos, mas há indícios de que a infecção pode ser causada pela ingestão ou injeção parenteral de sangue infectado (Berent \& Cooper 1998). Acredita-se também que a transmissão de M. haemofelis pode ocorrer principalmente por artrópodes hematófagos e também há a possibilidade de ocorrer por feridas causadas por mordeduras (Grace 2004). Ainda que possível, a transmissão vertical é considerada pouco importante (Page 2003).

0 diagnóstico geralmente é estabelecido pela demonstração do microorganismo em esfregaços de sangue periférico corados, cuja sensibilidade é bem reduzida, levando à subnotificação da ocorrência desses agentes (Hagiwara 2003). A Reação em Cadeia pela Polimerase (PCR) é o teste de escolha para o diagnóstico direto dessas infecções, principalmente em estudos epidemiológicos, visto que é uma ferramenta mais sensível e permite ainda fazer diferenciação entre as espécies de micoplasmas (Foley et al. 1998, Westfall et al. 2001).

Desse modo, o objetivo do presente trabalho foi detectar a infecção natural por M. haemofelis, 'Candidatus M. haemominutum' e 'Candidatus M. turicensis' em gatos domésticos do município de Belém, estado do Pará, através da PCR.

\section{MATERIAL E MÉTODOS}

\section{Comitê de Ética}

O presente projeto foi submetido ao Comitê de Ética no Uso de Animais da Universidade Estadual do Pará (CEUA-CCBS-UEPA) tendo sido aprovado sob o número de protocolo 06/12.

\section{Local e animais do estudo}

O local escolhido para o estudo foi a cidade de Belém $\left(01^{\circ} 27^{\prime}\right.$ $21^{\prime \prime}$ S, $48^{\circ} 30^{\prime} 16^{\prime \prime} \mathrm{W}$ ), capital do estado do Pará, que se localiza na região Norte do Brasil.
O grupo amostral foi constituído por 201 gatos domésticos, com idades variadas, independente de sexo ou raça e que foram divididos em três grupos. 0 grupo A foi composto por 101 animais errantes recolhidos pelo Centro de Controle de Zoonoses (CCZ) da Prefeitura Municipal de Belém; o grupo B foi composto por 62 animais clinicamente sadios que foram encaminhados a castração eletiva na Universidade Federal Rural da Amazônia (UFRA), enquanto que o grupo $\mathrm{C}$ foi composto por 38 animais portadores de alguma afecção clínica e que foram levados pelos seus proprietários para o atendimento clínico do Hospital Veterinário da UFRA.

\section{Coleta do material}

Durante o período de setembro de 2011 a abril de 2012 foi realizada a coleta de sangue dos gatos domésticos através da punção da veia cefálica, femoral ou jugular, após a contenção física.

\section{Reação em cadeia pela polimerase (PCR)}

Os DNAs foram extraídos a partir de $250 \mu \mathrm{L}$ do sangue de cada animal, utilizando-se o kit de extração de DNA genômico da Axygen Biosciences ${ }^{\circledR}$, seguindo as recomendações do fabricante.

Para a detecção de 'Candidatus Mycoplasma haemominutum' utilizou-se os iniciadores 1183F (5'-GCATAATGTGTC GCAATC - 3') e 1290R (5'- GTTTCAACTAGTACTTTCTCCC -3') que amplificam parte do gene 16SrRNA, segundo Foley et al (1998). A amplificação iniciou com $94^{\circ} \mathrm{C}$ por 7 minutos seguido por 35 ciclos de $94^{\circ} \mathrm{C}$ por 1 minuto, $53^{\circ} \mathrm{C}$ por 1 minuto, $72^{\circ} \mathrm{C}$ por 1 minuto seguido por uma extensão final a $72^{\circ} \mathrm{C}$ por 10 minutos.

Para a detecção de Mycoplasma haemofelis utilizou-se os iniciadores Hfelis-fl (5'- GACTTTGGTTTCGGCCAAGG -3') e Hfelis-r3 (5'- CGAAGTACTATCATAATTATCCCTC-3'), que amplificam parte do gene 16srRNA segundo Santos (2008). A amplificação iniciou com $94^{\circ} \mathrm{C}$ por 7 minutos seguido por 40 ciclos de $94^{\circ} \mathrm{C}$ por $1 \mathrm{mi}-$ nuto, $54^{\circ} \mathrm{C}$ por 1 minuto, $72^{\circ} \mathrm{C}$ por 1 minuto seguido por uma extensão final a $72^{\circ} \mathrm{C}$ por 10 minutos.

Para a detecção de 'Candidatus Mycoplasma turicensis' utilizou-se os iniciadores MT-FW (5'- GACTTTGGTTTCGGCCAAGG -3') e MT2-RV (5' - CGAAGTACTATCATAATTATCCCTC-3'), que amplificam parte do gene 16SrRNA segundo Santos (2008). A amplificação iniciou com $94^{\circ} \mathrm{C}$ por 7 minutos seguido por 40 ciclos de $94^{\circ} \mathrm{C}$ por 1 minuto, $54^{\circ} \mathrm{C}$ por 45 segundos, $72^{\circ} \mathrm{C}$ por 1 minuto seguido por uma extensão final a $72^{\circ} \mathrm{C}$ por 10 minutos.

A solução para a amplificação continha $5 \mu \mathrm{L}$ de DNA teste

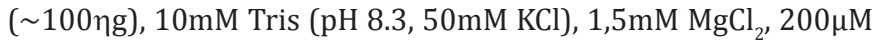
de cada nucleotídeo, $1 \mu \mathrm{M}$ de cada iniciador e 2 unidades de Taq DNA polimerase (Ludwig Biotec) totalizando um volume final de $25 \mu \mathrm{L}$.

As PCRs foram realizadas em um termociclador com gradiente (Multigene Labnet ${ }^{\circledR}$ ) e os produtos amplificados foram analisados por eletroforese horizontal em gel de agarose a 1,5\% corados com brometo de etídio $(0,5 \mu \mathrm{g} / \mathrm{mL})$. Em cada bateria de reações foi empregado o DNA de cada uma das três espécies de hemoplasmas pesquisados como controle positivo, como controle negativo foi empregado o DNA extraído de uma amostra de sangue de gato sabidamente negativo, e como controle de contaminação foi empregado água ultrapura em vez de DNA. 0 marcador de peso molecular utilizado foi de 100 pares de base (Ludwig Biotec). A visualização dos produtos amplificados foi realizada em sistema de fotodocumentação com transiluminador UV Quantum ST41000/26M.

Os amplicons foram purificados com o kit GFX DNA PCR and Gel Band Purification Kit (GE Healthcare), ligados ao vetor pGem-T Vector System (Promega) e inseridos em Escherichia coli Top 10 quimiocompetentes. O DNA dos clones recombinantes foi obtido por PCR, diretamente das colônias, e sequenciado com auxílio do 3500 XL Genetic Analyzer (Life Technologies) em conjunção 
com o BigDye ${ }^{\circledR}$ Terminator v3.1 Cycle Sequencing kit (Life Technologies), de acordo com as especificações do fabricante. Os amplicons foram individualmente seqüenciados com os iniciadores M13F/M13R e o programa BioEdit (Hall 2007) foi utilizado para o alinhamento das sequencias obtidas. A análise da identidade foi realizada através do programa BLAST disponível no National Center for Biotechnology Information (NCBI).

\section{Análise estatística}

0 teste exato de Fisher foi aplicado para analisar a influência do sexo e do grupo amostral sobre a detecção do DNA dos hemoplasmas nos animais estudados, com um nível de significância de 5\%. 0 Odds Ratio foi aplicado para avaliar as chances de se encontrar o DNA dos agentes estudados nos animais de cada grupo amostral. Para ambos os testes empregados utilizou-se o software Biostat $^{\circledR}$ versão 5.3.

\section{RESULTADOS}

A PCR utilizando-se os iniciadores $1183 \mathrm{~F}$ e $1290 \mathrm{R}$ gerou produtos de 192 pares de base que após o sequenciamento confirmou-se ser de 'Candidatus Mycoplasma haemominutum'. As reações utilizando-se os iniciadores Hfelis-fl e Hfelis-r3 geraram produtos de 393 pares de base de $M$. haemofelis e as reações com os iniciadores MT-FW e MT2-RV geraram produtos de $498 \mathrm{pb}$ de 'Candidatus Mycoplasma turicensis'. A análise no programa BLAST confirmou a especificidade das reações da PCR com identidade superior a $99 \%$ quando comparadas às obtidas neste estudo.

A frequência total de hemoplasmas foi de 19,9\% (40/201). 0 agente cujo DNA foi mais frequentemente detectado foi 'Candidatus M. turicensis' (12,93\%), seguido por 'Candidatus M. haemominutum' (7,96\%) e M. haemofelis $(1,49 \%)$. 0 DNA dos três agentes pesquisados foi detectado nos gatos domésticos dos Grupos A e C, enquanto que nos animais do Grupo B foi detectado somente o DNA de 'Candidatus M. turicensis' e 'Candidatus M. haemominutum' (Quadro 1). A coinfecção não foi um achado comum no presente estudo, sendo detectado em apenas 12,5\% (5/40) dos animais examinados. Os hemoplasmas mais frequentemente detectados foram 'Candidatus M. turicensis' (12,9\%) e 'Candidatus $M$. haemominutum' $(7,9 \%)$, seguido por $M$. haemofelis $(1,4 \%)$.

$\mathrm{Na}$ análise de risco verificou-se que as chances de se detectar o DNA de "Candidatus M. haemominutum" nos animais pertencentes ao grupo A foram 9,81 e 5,95 vezes maiores do que nos animais dos grupos $\mathrm{B}$ e $\mathrm{C}$, respectiva-

Quadro 1. Distribuição das amostras positivas para 'Candidatus Mycoplasma haemominutum' (CMH), Mycoplasma haemofelis (MH) e 'Candidatus Mycoplasma turicensis' (CMT) em função do grupo experimental e do sexo dos animais

\begin{tabular}{lcccc}
\hline \multirow{2}{*}{$\begin{array}{c}\text { Agente } \\
\text { etiológico }\end{array}$} & \multicolumn{3}{c}{ Grupos } & Total \\
\cline { 2 - 4 } & $\mathrm{A}$ & $\mathrm{B}$ & $\mathrm{C}$ & \\
\hline \multirow{2}{*}{ CMH } & $13,86 \%$ & $1,61 \%$ & $2,63 \%$ & $7,96 \%$ \\
& $(14 / 101)^{\mathrm{a}}$ & $(1 / 62)^{\mathrm{b}}$ & $(1 / 38)^{\mathrm{ab}}$ & $(16 / 201)$ \\
MH & $1,98 \%$ & $2,63 \%$ & $1,49 \%$ & \\
& $(2 / 101)$ & 0 & $(1 / 38)$ & $(3 / 201)$ \\
CMT & $15,84 \%$ & $3,22 \%$ & $21,05 \%$ & $12,93 \%$ \\
& $(16 / 101)^{\mathrm{a}}$ & $(2 / 62)^{\mathrm{b}}$ & $(8 / 38)^{\mathrm{a}}$ & $(26 / 201)$ \\
\hline
\end{tabular}

$\overline{a, b}$ Letras diferentes nas linhas indicam diferença estatística significativa.
Quadro 2. Distribuição das amostras positivas para 'Candidatus Mycoplasma haemominutum' (CMH), Mycoplasma haemofelis (MH) e 'Candidatus Mycoplasma turicensis' (CMT) em função do sexo dos animais

\begin{tabular}{lccc}
\hline Sexo & Machos & Fêmeas & Total \\
\hline CMH & $16,17 \%(11 / 68) \mathrm{a}$ & $3,75 \%(5 / 133)^{\mathrm{b}}$ & $7,96 \%(16 / 201)$ \\
MH & $2,94 \%(02 / 68)^{\mathrm{a}}$ & $0,75 \%(1 / 133)^{\mathrm{a}}$ & $1,49 \%(03 / 201)$ \\
CMT & $17,64 \%(12 / 68)^{\mathrm{a}}$ & $10,52 \%(14 / 133)^{\mathrm{a}}$ & $12,93 \%(26 / 201)$
\end{tabular}

$\overline{a, b}$ Letras diferentes nas linhas indicam diferença estatística significativa.

mente. As chances de se detectar o DNA de 'Candidatus M. turicensis' nos animais pertencentes ao grupo $C$ foram 1,4 e 8,0 vezes maiores do que nos animais dos grupos A e B, respectivamente. Já as chances de se encontrar o DNA de M. haemofelis nos animais do grupo $\mathrm{C}$ foram de 1,33 vezes maiores do que nos animais do grupo A. Em relação a influência do sexo dos animais sobre a infecção por hemoplasmas, foi detectada associação estatística apenas entre a infecção por 'Candidatus M. haemominutum' ( $p=0,0032)$ e os machos (Quadro 2).

\section{DISCUSSÃO}

Os hemoplasmas têm sido frequentemente detectados em esfregaço sanguíneo de gatos domésticos no Brasil, embora alguns trabalhos que investigam a ocorrência desses agentes apontem a PCR como o método de eleição para o diagnóstico direto, em virtude de sua maior sensibilidade e especificidade (Moraes et al. 2007, Biondo et al. 2009, Sykes 2010). Através da PCR pode-se detectar infecções com baixa carga parasitária comuns em estudos epidemiológicos onde os animais são selecionados de forma randômica (Foley et al. 1998).

Poucos trabalhos foram realizados com o objetivo de se determinar, através da PCR, a ocorrência em diferentes regiões brasileiras das três espécies de hemoplasmas de felinos atualmente reconhecidas. E, após extenso levantamento na literatura científica, observou-se que o presente trabalho é o primeiro a pesquisar, utilizando-se a PCR e com confirmação através sequenciamento do produto amplificado, a ocorrência de Mycoplasma haemofelis, 'Candidatus M. haemominutum' e 'Candidatus M. turicensis' em gatos domésticos da região Norte do Brasil (Santos 2008, Bartoli et al. 2012, Braga et al. 2012).

No presente estudo, a frequência de infecção por hemoplasmas está de acordo com o publicado por Souza e Almosny (2002), que relataram que a frequência de infecção por hemoplasmas na população felina no Brasil é estimada entre 5 e $23 \%$.

Taxas de infecção por Mycoplasma spp. inferiores às observadas no presente estudo foram detectadas através da PCR no Rio de Janeiro (12,1\%) (Macieira et al. 2009), em São Luís (14,5\%) (Braga et al. 2012) e em Jaboticabal (6,5\%) (Bartoli et al. 2012). Entretanto, há relatos de taxas de infecção por hemoplasmas superiores ao observado no presente estudo e que foram detectadas através do esfregaço sanguíneo, como os 38\% reportado por Mendes-de-Almeida (2004) no Rio de Janeiro e os 26,6\% por Miranda (2008) em Belém. Devido à dificuldade de visualização 
de hemoplasmas através do esfregaço sanguíneo, técnicas como a PCR e o sequenciamento devem ser empregados para evitar resultados falso-positivos e falso-negativos (Sykes 2010).

A análise comparativa da ocorrência dos hemoparasitos estudados demonstrou que não houve diferença entre a ocorrência de 'Candidatus M. haemominutum' e 'Candidatus M. turicensis' ( $\mathrm{p}=0,1030)$, contudo, esses agentes tiveram ocorrência superior a M. haemofelis $(\mathrm{p}=0,0022$ e $p=0,0001$, respectivamente). Outros estudos realizados no Brasil, e que utilizaram a PCR como ferramenta diagnóstica, também demonstraram a mesma tendência (Santos 2008, Bartoli et al. 2012, Braga et al. 2012).

$\mathrm{Na}$ análise comparativa entre as taxas de infecção por 'Candidatus M. haemominutum' e 'Candidatus M. turicensis', identificou-se o maior número de animais positivos oriundos do CCZ (Grupo A), indicando que o comportamento errante e o manejo dos animais, caracterizado pelo confinamento de vários gatos em um mesmo recinto, são fatores de risco para essas infecções. Em relação à taxa de infecção por 'Candidatus M. turicensis', não houve diferença significante entre os animais do Grupo A e do Grupo C, entretanto diferença estatística foi observada entre esses dois grupos e o grupo B, composto por animais sadios. A análise do odds ratio demonstrou que há maior chance de se detectar o DNA de 'Candidatus M. haemominutum' nos animais do grupo $\mathrm{A}$, enquanto que as maiores chances de se encontrar o DNA de 'Candidatus M. turicensis' e M. haemofelis foram maiores nos animais com distúrbios orgânicos e encaminhados ao veterinário (grupo $\mathrm{C}$ ).

A ocorrência mais frequente destes hemoplasmas, quando comparada com M. haemofelis pode se dar devido à diferença de patogenicidade entre esses agentes, pois 'Candidatus M. haemominutum' e 'Candidatus M. turicensis' são menos patogênicos do que $M$. haemofelis, ou seja, são mais bem sucedidos no estabelecimento do equilíbrio da relação parasito-hospedeiro, conseguindo manter a infecção sem desencadear doença grave, fato que é mais associado ao parasitismo por M. haemofelis (Foley et al. 1998, Sykes 2010). Na Espanha, Criado-Fornélio et al. (2003) realizaram um estudo com gatos sintomáticos e encontraram com maior frequência o DNA de M. haemofelis do que o DNA de 'Candidatus M. haemominutum'.

A associação entre anemia e infecção por esses hemoplasmas é controversa (Kewish et al. 2004, Ishak et al. 2007, Bauer et al. 2008), pois diferente do observado na infecção por M. haemofelis, a infecção por 'Candidatus M. haemominutum' tende a um quadro subclínico (Foley et al. 1998, Sykes 2010). Segundo Tasker (2010), em gatos naturalmente infectados por 'Candidatus M. turicensis', apenas os que apresentam coinfecção por M. haemofelis ou 'Candidatus M. haemominutum' tendem a apresentar valores de volume globular significativamente menores quando comparados a gatos sadios.

No que se refere à influência do sexo dos animais sobre as infecções por hemoplasmas, observou-se uma associação estatística apenas entre machos e a infecção por 'Candidatus M. haemominutum', assim como o reportado por Bauer et al. (2008). Já Santos (2008) associou gatos machos e com acesso a rua à maior prevalência da infecção por hemoplasmas de felinos. Sykes (2010) reportou episódios de hemoplasmose em animais machos após brigas, ratificando que hábitos de deambulação e brigas sejam fatores de risco para a infecção por hemoplasmas.

\section{CONCLUSÃO}

Foi possível concluir que as três espécies de hemoplasmas de felinos atualmente reconhecidas circulam entre os gatos domésticos do município Belém, sendo 'Candidatus Mycoplasma turicensis' e 'Candidatus M. haemominutum' mais frequentes que $M$. haemofelis.

\section{REFERÊNCIAS}

Bartoli C.P., André M.R., Seki M.C., Pinto A.A.M. \& Machado R.Z. 2012. Detection of hemoplasma and Bartonella species and co-infection with retroviruses in cats subjected to a spying/neutering program in Jaboticabal. Revta Bras. Parasitol. Vet. 21(3):219-223.

Bauer N., Balzer H.-J., Thüre S. \& Moritz A. 2008. Prevalence of feline haemotripic mycoplasmas in convenience samples of cats in Germany. J. Feline Med. Surg. 10:252-258.

Berent L.M., Messick J.B. \& Cooper S.K. 1998. Detection of Haemobartonella felis in cats with experimentally induced acute and chronic infections, using a polymerase chain reaction assay. Am. J. Vet. Res. 59(10):1215-20.

Biondo W.A., Santos A.P., Guimarães A.M.S., Vieira R.F.C., Vidotto O., Macieira D.B., Almosny N.R.P., Molento M.B., Timenetsky J., Morais H.A., Gonzales F.H.D. \& Messick J.B. 2009. A review of the occurrence of hemoplasmas (hemotropic mycoplasmas) in Brazil. Revta Bras. Parasitol. Vet. 18(3):1-7.

Braga M.S.O., André M.R., Freschi C.R., Teixeira M.C.A. \& Machado R.Z. 2012. Molecular detection of hemoplasma infection among cats from São Luis Island, Maranhão, Brazil. Braz. J. Microbiol. 43(2):569-575.

Foley J.E., Harrus S., Poland A., Chomel B. \& Pedersen N.C. 1998. Molecular, clinical, and pathologic comparison of two distinct strains of Haemobartonella felis in domestic cats. Am. J. Vet. Res. 59(12):1581-1588.

Grace S.F. 2004. Anemia, p.8-13. In: Gary D.O. (Ed.), Paciente Felino. $2^{\underline{a}}$ ed. Manole, São Paulo.

Hagiwara M.K. 2003. Anemia, p.15-24. In: Souza H.J. (Ed.), Coletâneas em Medicina e Cirurgia Felina. L.F. Livros, Rio de Janeiro.

Ishak A.M., Rodecki S. \& Lappin M.R. 2007. Prevalence of Mycoplasma haemofelis, Candidatus Mycoplasma haemominutum, Bartonella species, Ehrlichia species, and Anaplasma phagocytophilium DNA in the blood of cats with anemia. J. Feline Med. Surg. 9:1-7.

Kewish K.E., Appleyard G.D., Myers S.L., Kidney B.A. \& Jackson M.L. 2004. Mycoplasma haemofelis and Mycoplasma haemominutum detection by polymerase chain reaction in cats from Saskatchewan and Alberta. Can. Vet. J. 45:749-752.

Macieira D.B., Menezes R.C.A.A., Damico C.B., Almosny N.R.P. \& Messick J.B. 2009. Uso da técnica de southern blot/hibridização associada à reação em cadeia da polimerase para aumentar a sensibilidade no diagnóstico das infecções por hemoplasmas em gatos domésticos. Revta Bras. Parasitol. Vet. 18(Supl.):1-6.

Mendes-de-Almeida F., Faria M.C.F., Branco A.S., Serrão M.L., Souza A.M., Almosny N., Chame M. \& Labarthe N. 2004. Sanitary conditions of a colony of urban feral cats (Felis catus Linnaeus, 1758) in a zoological garden of Rio de Janeiro, Brazil. Revta Inst. Med. Tropical, São Paulo, 46(5):269-274.

Messick J.B. 2004. Hemotropic mycoplasmas (hemoplasmas): a review and new insights into pathogenic potential. Vet. Clin. Pathol. 33:2-13.

Miranda C.F. 2008. Prevalência de Mycoplasma haemofelis (Hemobartonella felis) em gatos domésticos (Felis catus Linaeus, 1759) na região metropolitana de Belém. Trabalho de conclusão de curso (especialização em clínica médica de pequenos animais), Universidade Castelo Branco, Belém, PA. 39p. 
Moraes H.A., Guimarães A.M.S., Vidotto O., Baumman A., Biondo A.W. \& Messick J.B. 2007. Co-infection with Mycoplasma haemofelis and Candidatus Mycoplasma haemominutum in three cats from Brazil (short communication). J. Feline Med. Surg. 9:518-520.

Page R.L. 2003. Hematologia/oncologia: hemácias, leucócitos e plaquetas, Seção 3, p.165-183. In: Bichard S.J. \& Sherding R.G. (Eds), Manual Saunders, Clínica de Pequenos Animais. $2^{\text {nd }}$ ed. W.B. Saunders, Philadelphia.

Santos A.P. 2008. Infecção por hemoplasmas felinos na região de Porto Alegre, RS, Brasil. Tese de Doutorado em Ciências Veterinária, Universidade Federal do Rio Grande do Sul, Porto Alegre. 162p.

Souza A.M. \& Almonny N.R.P. 2005. Hemobartonelose em pequenos animais domésticos e como zoonose, p.90-101. In: Almonsy Nadia R.P. (Ed.), Hemoparasitoses em Pequenos Animais Domésticos e como Zoonoses. L.F. Livros de Veterinária, Rio de Janeiro.
Sykes J.E. 2003. Feline hemotropic mycoplasmosis (feline hemobartonellosis). Vet. Clin. North Am., Small Anim. Pract. 33(4):773-787.

Sykes J.E. 2010. Feline hemotropic mycoplasmas. Vet. Clin. North Am., Small Anim. Pract. 40:157-1170.

Tasker S. 2010. Haemotropic mycoplasmas: what's the real significance in cats? J. Feline Med. Surg. 12:369-381.

Willi B., Boretti F.S., Cattori V., Tasker S., Meli M.L., Reusch C., Lutz H. \& Hofmann-Lehmann R. 2005. Identification, molecular characterization and experimental transmission of a new hemoplasma isolate from a cat with hemolytic anemia in Switzerland. J. Clin. Microbiol. 43(6):2581-2585.

Westfall D.S., Jensen A.W., Reagan W.J., Radecki S.V. \& Lappin M.R.. 2001. Inoculation of two genotypes of Haemobartonella felis (California and Ohio variants) to induce infection in cats and the response to treatment with azithromycin. Am. J. Vet. Res. 62:687-691. 